\title{
ON SQUARE ROOTS AND LOGARITHMS OF SELF-ADJOINT OPERATORS
}

\author{
by C. R. PUTNAM
}

(Received 17th February, 1958)

All operators considered in this paper are bounded and linear (everywhere defined) on a Hilbert space. An operator $A$ will be called a square root of an operator $B$ if

$$
A^{2}=B \text {. }
$$

A simple sufficient condition guaranteeing that any solution $A$ of (1) be normal whenever $B$ is normal was obtained in [1], namely: If $B$ is normal and if there exists some real angle $\theta$ for which $\operatorname{Re}\left(A e^{i \theta}\right) \geqslant 0$, then (1) implies that $A$ is normal. Here, $\operatorname{Re}(C)$ denotes the real part $\underline{1}\left(C+C^{*}\right)$ of an operator $C$.

The object of the present note is to use the above result to generalize the well-known fact that a (self-adjoint) non-negative operator has a unique non-negative square root (cf. [3], p. 256, also [2], p. 725) and to obtain a certain uniqueness theorem for logarithms of positive self-adjoint operators. The following will be proved :

(I) If $B$ is a non-negative self-adjoint operator, and if $A$ is any solution of (1) ( $A$ not assumed to be self-adjoint or even normal) satisfying $\operatorname{Re}(A) \geqslant 0$, then necessarily $A$ is the (unique) nonnegative self-adjoint square root of $B$.

(II) If $A$ is a logarithm of a positive self-adjoint operator $B=\int \lambda d E$, so that $e^{A}=B(>0)$, and if

$$
\|A\| \leqslant 2 \log 2,
$$

then necessarily $A$ is the self-adjoint operator

$$
A=\int \log \lambda d E \quad(\log \lambda \text { real })
$$

The proof of (I) follows from an application of the italicized assertion in the first paragraph. For, since $B$ is self-adjoint and hence normal, $A$ is normal. Since the square of any number in the spectrum of $A$ is in the spectrum of $B$, it follows that the spectrum of $A$ is real. Therefore $A$ is self-adjoint and, in view of the assumption $\operatorname{Re}(A) \geqslant 0$, is non-negative (hence uniquely determined). This completes the proof of (I).

In order to prove (II), it will first be shown that

$$
e^{A / 2}=B^{1 / 2}
$$

where $B^{1 / 2}$ denotes the (unique) positive square root of $B$. To this end, note that

$$
\left\|e^{A / 2}-I\right\| \leqslant e^{\|A\| / 2}-1 \leqslant 1
$$

the second inequality following from (2). Hence $\operatorname{Re}\left(e^{A / 2}\right) \geqslant 0$ and (4) now follows from (I).

Since the inequality (2) holds also if $A$ is replaced by $A / 2^{n}$ for $n=1,2, \ldots$, it follows that $e^{A / 2^{n}}=B^{1 / 2^{n}}$ for $n=0,1,2, \ldots$. Consequently, $e^{r A}=H^{r}$ for any rational number of the form $r=m / 2^{n}(n=0,1,2, \ldots ; m=0, \pm 1, \pm 2, \ldots)$ and hence, by continuity,

$$
e^{\prime \mathbf{A}}=B^{t}=\int \lambda^{t} d E
$$


for every real $t$. A differentiation with respect to $t$ of this (operator) identity yields

$$
A e^{i \Delta}=\left(\int \log \lambda d E\right) B^{t}
$$

hence for $t=0$, the relation (3), at least for some determination of $\log \lambda$. But $\|A\| \geqslant|\log \lambda|$ for every $\lambda$ in the (real) spectrum of $B$ and so relation (2) implies that $\log \lambda$ is real. This completes the proof of (II).

. This research was supported by the United States Air Force under Contract No. AF 18 (603) -139.

\section{REFERENCES}

1. C. R. Putnam, On square roots of normal operators, Proc. Amer. Math. Sor., 8 (1957), $768-769$.

2. F. J. Wecken, Zur Theorie linearer Operatoren, Math. Annalen 110 (1935), 722-725.

3. A. Wintner, Ueber das Aequivalenzproblem beschränkter hermitescher Formen, Math.Z., 37 (1933), 254-263.

Purdue Untverstty

LAFAYETTE, INDIANA

U.S.A. 